\title{
Predictions and insights for the abdominal aortic aneurysm
}

\section{Thomas Kotsis}

Head of Vascular Unit, Aretaieion University Hospital, Medical School, National and Kapodistrian University of Athens, Athens, Greece

Correspondence to: Dr Thomas Kotsis. Head of Vascular Unit, Aretaieion University Hospital, Medical School, National and Kapodistrian University of Athens, 76 Vas. Sophias Av., 11526 Athens, Greece. Email: kotsisth@otenet.gr.

Provenance: This is an invited Editorial commissioned by the Section Editor Lei Zhang (Department of Vascular Surgery, Changhai Hospital, Second Military Medical University, Shanghai, China).

Comment on: MA3RS Study Investigators. Aortic Wall Inflammation Predicts Abdominal Aortic Aneurysm Expansion, Rupture, and Need for Surgical Repair. Circulation 2017;136:787-97.

Submitted Aug 06, 2018. Accepted for publication Oct 02, 2018.

doi: $10.21037 /$ jtd.2018.10.15

View this article at: http://dx.doi.org/10.21037/jtd.2018.10.15

Modern vascular surgery imposes its principal preventive role worldwide, by focusing on two arterial diseases, carotid artery stenosis and aortic aneurysms. Both vascular disorders may result in a debilitating or life-threatening situation. There is a tremendous scientific interest about the vulnerable asymptomatic plaques and the aortic aneurysm with imminent risk of rupture; thousands of scientific hours have been consumed in order to identify which asymptomatic carotid plaque is the potent source of emboli for an ischemic stroke, as well as to detect which aortic aneurysm is going to rupture. In an attempt to draw a universal cut-off line for asymptomatic diseases, the degree of carotid stenosis remains an acceptable criterion for the carotid disease, although the intervention in an asymptomatic carotid stenosis is often debated by many investigators. Similarly, for aortic aneurysms, the maximum diameter of the aneurysm along with the aneurysm growth rate still define globally accepted thresholds for intervention (1).

Nevertheless, many factors associated either with the carotid disease, or with the aneurismal aorta, are being investigated and with parallel assessment of the risks, aim at fine-tuning a personalized indication for surgery, certainly before the manifestation of the sound complications from both diseases. This endeavor consists of an intense hunting for the so-called markers, as paraclinical indices of the altered physiology; the ideal marker must be highly proportional to the process of the disease or has to reflect specific conditions with major clinical impact, such as aneurysm expansion or rupture (2). If the markers of such a quality exist, and if they can be identified by current modalities, they may alter the contemporary recommendations concerning the intervention indications, by precisely predicting a forthcoming disaster. Like in all scientific fields, following the initial related and crosschecking evidences, a hypothesis is built and through observational, or experimental studies, expectations for important reliable markers are hopefully created.

In particular, for the abdominal aortic aneurysm (AAA), with an incidence of more than $3-5 \%$ in general population, we have only a scarce bit of information about the true pathophysiology of an aortic dilatation. There is no doubt that an aneurysm is a disease mainly of the tunica media (and the adventitia) of the artery, that finally obeys to the simplified law of Laplace (bubble law) and ruptures. As 95\% of all the aortic aneurysms are infrarenal, investigations are focused on this entity, trying to reveal the causes for this nature's predilection with lethal consequences (dilatationexpansion, and rupture with $>90 \%$ mortality).

The two poles for an aortic aneurysm pathology are: the disease of the proper aortic wall, and the stress acted on the wall; they both contribute in a rupture, that occurs at the weakest site of the aortic wall with/or where the most intense wall stress is exerted. The weakness of the infrarenal aortic wall is determined by several factors such as genes, elastin/collagen imbalance, relative lack of vasa vasorum, metalloproteinase activity, pathogenic microorganism presence (e.g., Chlamydia) which impacts immune activity, and inflammation $(3,4)$. The aortic wall undergoes a continuous dynamic remodeling in order to withstand, and promote the cardiac pulse wave and as a response to injury; 
aging with a subsequent gene regulation derangement results in a diseased aortic wall leading to an aneurysm formation; gene erratic regulation is obvious in patients with connective tissue disorders where due to the aortic wall disease, aneurysms are present in young age; the incidence of inherited aneurysm formation due to familial gene disorders is up to $20 \%$ for first order relatives. Aortic wall deterioration may be induced by atherosclerosis, but the latter is rather a coincidence than a determining factor for infrarenal aortic dilatation, though wall ischemic changes due to vasa vasorum occlusion in obstructive arteriopathy have been observed.

In addition to the aortic wall weaknesses, the shape of the aorta is an important parameter involved in aortic dilatation or expansion, as the blood, an incompressible fluid, propelled by cardiac thrust, interacts strongly with a tortuous aortic wall. In the 1830 , Chelius in his Handbook of Surgery, postulates that arterial curves promote aneurysm formation (5). Newer studies state that there is an anisotropic energy distribution inside the aorta, in longitudinal and in transverse direction (2); it also seems that the aorta firstly elongates and then dilates (6). Peak wall stresses in $3 \mathrm{D}$ models estimated by finite element analysis, can explain the rupture in specific aortic loci as a phenomenon not necessarily dependent exclusively on local diameter or wall thickness. Additionally, the firm ring outside the inter-renal aortic portion combined with the aortic pulse counterwave from the aortic bifurcation expose the contained infrarenal aortic section to a forceful fluidstructure interaction. This reflective wave is amplified, and it strains the wall with a greater intensity in case of aorticcommon iliac surface area mismatch, and in augmented aortic stiffness. The aortic wall, already defected or not, reacts in this new increased tension, with an increase of the surface (enlargement), to ameliorate the local exerted pressure; this process leads to the ongoing expansion in an equilibrium of horror between wall tension (depended from the diameter) and matrix components of remodeling; that is the reason we meet enormous aneurysms without rupture. When the destabilizing components like the metalloproteases (MMPs) dominate over their inhibitors (TIMMPs), down regulation of the structural components takes place, resulting in a decreased wall strength, and the aortic wall ruptures. Body size and habitus, male gender, epidemiological risk factors like smoking, chronic obstructive pulmonary disease (COPD), benign prostatic hyperplasia (BPH), and other, act in combination inflicting wall injury, or to expand or promote a rupture of the
AAA (7). This multifactorial synergy obviously transmits signals through a biochemical pathway. The detection of such biomarkers in the plasma (MMPs) currently it is not very promising, and the markers are not anatomically specific for a certain diseased section of the vasculature. However, due to the present advanced imaging capabilities with an excellent resolution and diminution of study time, the combination of the imaging findings with specific molecular metabolic activity shines light on a long route to prediction for AAAs (8-10). This is currently attempted with positron emission tomography (PET), with fusion techniques with computed tomography (CT) or magnetic resonance imaging (MRI), and recently by using ultra small superparamagnetic particles of iron oxide (USPIOs) with an MRI scan (11,12). A convenient metabolic pathway, which is evident in the aortic wall, is inflammation where infiltration of the tunica media by lymphocytes and macrophages has been histologically proven. The activity of macrophages may be revealed, using cell-specific PET tracers such as $18 \mathrm{~F}$-fluorodeoxyglucose (18F-FDG) which is a glucose analogue or others, however imaging of AAA is accompanied by a low specificity due to its multifactorial nature; thus, this method is not recommended for a routine use on clinical basis $(12,13)$. Recent data from a welldesigned prospective multicenter study, the MA3RS with a cohort of 342 patients with AAAs $>4.0 \mathrm{~cm}$ and a followup of two years concluded that USPIO-enhanced MRI can predict the rate of aneurysm growth and clinical outcome, but this modality cannot be accepted as an independent predictor of aneurysm expansion or clinical outcomes in a model incorporating known clinical factors; the authors of MA3RS study state that USPIO enhancement appeared to be greater in those with emergent AAA related events, including aortic aneurysm rupture although this has to be supported by more studies (14). However, MA3RS study, by establishing a reliable method to detect at least one metabolic pathway in AAAs, opens a field for medical treatment of aortic wall inflammation through molecular vectoring, and most importantly, through USPIO-enhanced MRI the result of the respective medical treatment can be assessed. Additionally, as the use of USPIO is not associated with the risk of nephrogenic sclerosis like gadolinium, and due to its long-lasting blood pool enhancement, it may be used in MR studies that require detailed and/or long-lasting vessel imaging like MR angiography, though it can provoke immune reactions.

In a holistic approach, every single study concerning the physiology of AAAs is welcome; in clinical praxis prediction 
is required for the individualization of treatment. However, the scientific challenge for AAAs is, following an early detection of an aorta with a propensity for dilatation or with a small aneurysm-U/S studies consist a realistic methodto identify with the proper detecting molecules all the metabolic pathways of the disease, by tracking alterations in molecular level at the microenvironment of the aortic tissue (15). Then, by using the same detectors as vectors, or through other medical solutions, we have to modify the aortic wall by remodeling and enhancing the up regulation of the strengthening matrix components, with the aim to interrupt and reverse this hostile biological irregularity at an early stage. Until then, we need early predictors to tell us when to use with safety, our open or endovascular mechanical solutions (16), in the hope that we change the natural history of the disease.

\section{Acknowledgements}

None.

\section{Footnote}

Conflicts of Interest: The authors have no conflicts of interest to declare.

\section{References}

1. Chaikof EL, Dalman RL, Eskandari MK, et al. The Society for Vascular Surgery practice guidelines on the care of patients with an abdominal aortic aneurysm. J Vasc Surg 2018;67:2-77.e2.

2. Choke E, Cockerill G, Wilson WR, et al. A review of biological factors implicated in abdominal aortic aneurysm rupture. Eur J Vasc Endovasc Surg 2005;30:227-44.

3. Wassef M, Baxter BT, Chisholm RL, et al. Pathogenesis of abdominal aortic aneurysms: a multidisciplinary research program supported by the National Heart, Lung, and Blood Institute. J Vasc Surg 2001;34:730-8.

4. Jalalzadeh $\mathrm{H}$, Indrakusuma R, Planken RN, et al. Inflammation as a Predictor of Abdominal Aortic Aneurysm Growth and Rupture: A Systematic Review of Imaging Biomarkers. Eur J Vasc Endovasc Surg

Cite this article as: Kotsis T. Predictions and insights for the abdominal aortic aneurysm. J Thorac Dis 2018;10(11):59825984. doi: 10.21037/jtd.2018.10.15
2016;52:333-42.

5. Chelius MJ. Handbuch der Chirurgie. 1830. Available online: http://www.mdz-nbn-resolving.de/urn/resolver. pl?urn=urn:nbn:de:bvb:12-bsb11081586-8

6. Michineau S, Dai J, Gervais M, et al. Aortic length changes during abdominal aortic aneurysm formation, expansion and stabilisation in a rat model. Eur J Vasc Endovasc Surg 2010;40:468-74.

7. Lo RC, Lu B, Fokkema MT, et al. Relative importance of aneurysm diameter and body size for predicting abdominal aortic aneurysm rupture in men and women. J Vasc Surg 2014;59:1209-16.

8. Forsythe RO, Newby DE, Robson JM. Monitoring the biological activity of abdominal aortic aneurysms Beyond Ultrasound. Heart 2016;102:817-24.

9. Baliyan V, Verdini D, Meyersohn NM. Noninvasive aortic imaging. Cardiovasc Diagn Ther 2018;8:S3-18.

10. McBride OM, Joshi NV, Robson JM, et al. Positron Emission Tomography and Magnetic Resonance Imaging of Cellular Inflammation in Patients with Abdominal Aortic Aneurysms. Eur J Vasc Endovasc Surg 2016;51:518-26.

11. Lee H, Paeng JC, Kim KH, et al. Correlation of FDG PET/CT Findings with Long-Term Growth and Clinical Course of Abdominal Aortic Aneurysm. Nucl Med Mol Imaging 2018;52:46-52.

12. Conlisk N, Forsythe RO, Hollis L, et al. Exploring the Biological and Mechanical Properties of Abdominal Aortic Aneurysms Using USPIO MRI and Peak Tissue Stress: A Combined Clinical and Finite Element Study. J Cardiovasc Transl Res 2017;10:489-98.

13. Richards JM, Semple SI, MacGillivray TJ, et al. Abdominal aortic aneurysm growth predicted by uptake of ultrasmall superparamagnetic particles of iron oxide: a pilot study. Circ Cardiovasc Imaging 2011;4:274-81.

14. MA3RS Study Investigators. Aortic Wall Inflammation Predicts Abdominal Aortic Aneurysm Expansion, Rupture, and Need for Surgical Repair. Circulation 2017;136:787-97.

15. Hu C, Zhu K, Li J, et al. Molecular targets in aortic aneurysm for establishing novel management paradigms. J Thorac Dis 2017;9:4708-22.

16. Scharrer-Pamler R, Kotsis T, Kapfer X, et al. Endovascular stent-graft repair of ruptured aortic aneurysms. J Endovasc Ther 2003;10:447-52. 